\title{
Sasa veitchii extracts suppress acetaminophen-induced hepatotoxicity in mice
}

\author{
Hiroki Yoshioka ${ }^{1 *}$, Haruki Usuda ${ }^{2}$, Hirohisa Fujii ${ }^{1}$ and Tsunemasa Nonogaki ${ }^{*}$
}

\begin{abstract}
Background: The aim of this study was to investigate the therapeutic effects of a Sasa veitchii leaf extract (SE) on acetaminophen (APAP)-induced hepatotoxicity.

Methods: Seven-week-old male ddY mice were orally administered SE or saline $(0.2 \mathrm{~mL})$ once a day for a week. Twenty-four hours after the last pretreatment, the mice were intraperitoneally injected with $550 \mathrm{mg} / \mathrm{kg}$ APAP or saline under fasting conditions. The mice from each group were euthanized and bled for plasma analysis 2, 6, 24, and $72 \mathrm{~h}$ after the injection.

Results: We found that pretreatment with SE significantly decreased hepatic injury markers (i.e., alanine aminotransferase and aspartate aminotransferase), oxidative stress (malondialdehyde and glutathione level), inflammatory cytokines, histological damage, c-jun N-terminal kinase activation, and receptor-interacting protein-1 activation. Further, SE pretreatment decreased Cyp2e1 expression and increased total antioxidant capacity in the liver.

Conclusion: Our findings demonstrate that prophylactic SE treatment protects mice from APAP-induced hepatotoxicity through modulation of Cyp2e1 expression and antioxidant capacity.
\end{abstract}

\section{Background}

Bamboo leaves have been extensively used in folk medicine as an antifebrile and antihypertensive medication for centuries $[1,2]$. In addition, in Japan, due to their antimicrobial activity, bamboo leaves have been used to wrap sushi sheets to protect against bacterial spoilage. Recently, extracts of bamboo leaves were reported to have multiple biological activities including antioxidant activity and cancer prevention [3, 4]. Furthermore, previous studies have demonstrated the antitumor [5], antioxidant [1, 6], antiviral [7], anti-inflammatory [8], and anti-allergic [9] activities of the extract.

The liver is most vulnerable to attack by chemical toxic agents since it is one of the most internal organs with multiple functions such as detoxification and protein synthesis $[10,11]$. Therefore, liver diseases are among the most serious health problems worldwide. Our previous investigation found that Sasa veitchii leaf

\footnotetext{
* Correspondence: h-yoshioka@kinjo-u.ac.jp; tunenono@kinjo-u.ac.jp ${ }^{1}$ College of Pharmacy, Kinjo Gakuin University, 2-1723 Omori, Moriyamaku, Nagoya, Aichi 463-8521, Japan

Full list of author information is available at the end of the article
}

extract (SE) prevented carbon tetrachloride $\left(\mathrm{CCl}_{4}\right)$-induced hepatotoxicity in mice [12], suggesting that SE might maintain liver homeostasis. Although $\mathrm{CCl}_{4}$ is commonly used in animal models to study chemical toxin-induced liver injury $[13,14]$, exposure to $\mathrm{CCl}_{4}$ does not reflect a real-life scenario. The most common etiologies of liver damage in real life are acute viral hepatitis A and B, drug-related liver injury, and the alcohol-acetaminophen syndrome (AAS). In terms of drug-related liver injury, acetaminophen (APAP) is well known. In addition, the main cause of acute liver failure in industrialized countries is APAP overdose. APAP is recognized as a popular analgesic and antipyretic drug at therapeutic doses. However, APAP can cause severe liver injury in animals and humans through its side effects [15]. The main mechanism of APAP-induced hepatotoxicity has been studied in detail [16-19] and occurs in multiple steps. First, APAP is majority metabolized by Cyp2e1 and minority by Cyp1a2. Cyp2e1 is the main Cyp enzyme that bio-activates APAP at low doses $[16,20]$. In contrast, at a high dose, Cyp1a2 was shown to contribute to the bio-activation and toxicity of APAP [21]. Cyp- 
produced $N$-acetyl-p-benzoquinone imine (NAPQI) depletes glutathione (GSH) and covalently binds to proteins. Loss of GSH then permits increased formation of reactive oxygen and nitrogen species. As a result, oxidative stress is increased, leading to alterations in calcium homeostasis and initiation of signal transduction responses causing mitochondrial permeability transition. Mitochondrial permeability transition causes additional oxidative stress and inhibits ATP synthesis. Insufficient ATP triggers cell necrosis. Peripheral to these essential events, a number of inflammatory mediators such as cytokines and chemokines are induced that can modify toxicity.

Currently, the best antidote against APAP-induced hepatic injury is $\mathrm{N}$-acetylcysteine (NAC). NAC effectively minimizes APAP-induced toxicity when injected within a short time after APAP overdose [22]. However, clinical studies have revealed that injection of NAC often causes undesirable side effects [23]. Therefore, a need exists for compounds or chemicals that offer maximum protection against APAP-induced hepatotoxicity without NAC's side effects. The search for new drugs and novel therapeutic intervention strategies increasingly includes testing plant extracts and other natural products. It is generally accepted that natural products have multiple activities including anticancer and antioxidant [24, 25]. Owing to the critical and urgent need to explore the therapeutic potential of natural products and other compounds for prevention and treatment of hepatotoxicity, we evaluated the protective effects of SE against APAPinduced hepatic injury.

\section{Methods}

\section{Animal experimentation}

Six-week-old male ddY mice were purchased from Japan SLC (Shizuoka, Japan). They were housed under standard conditions of controlled temperature $\left(24 \pm 1{ }^{\circ} \mathrm{C}\right)$, humidity $(55 \pm 5 \%)$, and light (12-h/12-h light/dark cycle) and provided food and water ad libitum. All experiments were approved by the Institutional Animal Care and Experimentation Committee of Kinjo Gakuin University (No. 129).

\section{Preparation of SE}

SE was obtained from Sunchlon Co., Ltd. (Nagano, Japan) and was prepared using the method described previously $[9,12]$. One milliliter of SE was obtained from $2.82 \mathrm{~g}$ of $S$. veitchii leaves according to the company's data. The diluted SE solution contained minerals, carbohydrates, fat, protein, and silicic acid, as shown in Table 1.

\section{Treatment protocol}

Mice were pretreated with $0.2 \mathrm{~mL}$ of $\mathrm{SE}$ or saline as a control once a day for a week by oral gavage. Mice from
Table 1 Component of SE

\begin{tabular}{ll}
\hline Component & $\mathrm{mg} / \mathrm{mL}$ \\
Cu-chlorophyll & 250.00 \\
Carbohydrate & 8.17 \\
Fat & 3.58 \\
Protein & 3.08 \\
Ash content & 0.83 \\
Silicic acid & 0.42 \\
Water & 940.80 \\
Minerals & $\mu \mathrm{g} / \mathrm{mL}$ \\
$\mathrm{Na}$ & 2250.00 \\
$\mathrm{Fe}$ & 5.67 \\
$\mathrm{Zn}$ & 1.83 \\
$\mathrm{Cu}$ & 733.33 \\
$\mathrm{Mn}$ & 0.18 \\
$\mathrm{Ca}$ & 11.67 \\
$\mathrm{Mg}$ & 15.00 \\
$\mathrm{~K}$ & 86.67 \\
$\mathrm{P}$ & 18.33 \\
\hline
\end{tabular}

each group were fasted for $16 \mathrm{~h}$, and $550 \mathrm{mg} / \mathrm{kg}$ APAP dissolved in saline/polyethylene glycol emulsion or vehicle only (as a control) was i.p. injected at $10 \mathrm{~mL} / \mathrm{kg}$ body weight. The mice were euthanized and bled to obtain plasma 2, 6, 24, or $72 \mathrm{~h}$ following the injection. The resulting plasma samples were stored at $-80{ }^{\circ} \mathrm{C}$. The livers were harvested and stored at $-80{ }^{\circ} \mathrm{C}$ or fixed in $15 \%$ phosphate-buffered neutral formalin ( $\mathrm{pH} 7.4)$.

\section{Plasma biochemical analysis}

Plasma activities of alanine aminotransferase (ALT) and aspartate aminotransferase (AST) were measured using the Transaminase CII kit (Wako Pure Chemical, Osaka, Japan) according to the manufacturer's instructions, as previously described [26]. Plasma levels of tumor necrosis factor (TNF)- $\alpha$ (eBioscience, San Diego, CA, USA) were determined using commercially available ELISA kits, according to the manufacturer's instructions. For relative quantification, calibration curves were prepared using standard solutions.

\section{Histopathological findings}

For histological analysis, a portion of the left liver lobe from each animal was perfused with $15 \%$ phosphate-buffered neutral formalin ( $\mathrm{pH}$ 7.4), dehydrated, and embedded in paraffin. Embedded tissues were sectioned at $4 \mu \mathrm{m}$.

For immunohistochemistry, paraffin-embedded sections were deparaffinized and rehydrated in a grated ethanol series. After antigen retrieval by proteinase $\mathrm{K}$ (Wako Pure chemical) and quenching of endogenous 
peroxidase by hydrogen peroxide (Wako Pure chemical), sections were incubated with rat anti-TNF $\alpha$ monoclonal antibody (Biolegend, San Diego, CA, USA) as primary antibodies (1:250 dilution) at $4{ }^{\circ} \mathrm{C}$ for overnight. The color reaction was developed with peroxidase-conjugated antirat IgG (Sigma-Aldrich, St. Louis, MO, USA) as secondary antibody (1:750 dilution). In addition, sections were counterstained with hematoxylin.

For the detection of nuclear DNA strand breaks, paraffin-embedded sections were stained with the terminal dUTP nick-end labeling (TUNEL) technique using an in situ apoptosis detection kit (Takara Bio, Shiga, Japan) according to the manufacture's protocols. Sections were counterstained with hematoxylin.

For hematoxylin and eosin (H\&E) stain, we previously described [26].

\section{Measurement of malondialdehyde and GSH levels in the liver}

Total malondialdehyde (MDA) levels and total antioxidant capacity in the liver were examined using a colorimetric thiobarbituric acid reactive substances microplate assay kit and a colorimetric total antioxidant power assay kit, respectively (Oxford Biochemical Research, Oxford, MI, USA), according to the manufacturer's protocols, as previously described [27]. Hepatic GSH levels were measured using GSSG/GSH quantification kit (Dojindo Laboratories, Kumamoto, Japan) according to the manufacturer's instructions and as previously described [28].

\section{Western blot analysis}

0.1 -g liver sections were homogenized with $900 \mu \mathrm{L}$ icecold phosphate-buffered saline containing protease inhibitor (Nacalai Tesque, Kyoto, Japan) and centrifuged at $18,000 \mathrm{~g}$ for $20 \mathrm{~min}$ at $4{ }^{\circ} \mathrm{C}$. The resulting supernatant for each sample was collected and protein level determined using the BCA protein kit (Nacalai Tesque). Protein samples $(40 \mu \mathrm{g})$ were subjected to sodium dodecyl sulfate-polyacrylamide electrophoresis on a $10 \%$ gel and transferred to a polyvinylidene difluoride membrane. Rabbit anti-c-jun N-terminal kinase (JNK) polyclonal antibody, rabbit anti-phospho-JNK monoclonal antibody
(Cell Signaling Technology, Beverly, MA, USA), and rabbit anti-Cyp2e1 polyclonal antibody (Enzo Life Sciences, NY, USA) were used as primary antibodies (1:1000 dilution) for immunoblotting. A peroxidase-conjugated anti-rabbit IgG (Cell Signaling Technology) was used as secondary antibody (1:3000 dilution). Mouse anti-receptor-interacting protein (RIP)-1 monoclonal antibody (Santacruz, California, CA, USA) and mouse anti- $\beta$-actin monoclonal antibody (MBL, Aichi, Japan) were used as primary antibody (1:1000 dilution) for immunoblotting. A peroxidase-conjugated anti-mouse IgG (MBL) was used as secondary antibody (1:5000 dilution). The immunoreactive bands were visualized with the ECL system (BioRad, Hercules, CA, USA).

\section{Isolation of total RNA and qRT-PCR assay}

Total RNA was extracted from 0.1-g liver sections using the ISOGEN II kit (Nippon Gene, Tokyo, Japan). qRTPCR was performed with One-Step SYBR PrimeScript PLUS RT-PCR kit (Perfect Real Time) (Takara Bio) using an Applied Biosystems 7300 system (Applied Biosystems, Foster City, CA, USA). PCR conditions were as previously described [28]. Primer pairs are shown in Table 2. Relative expression of each messenger RNA (mRNA) was determined using the standard curve method. The amount of each target mRNA quantified was normalized against that of GAPDH-encoding mRNA.

\section{Statistical analysis}

Statistical significance of the differences between two groups was estimated using a two-tailed Student $t$ test. Multiple comparisons were made by one-way analysis of variance with post hoc Tukey-Kramer's test. All statistical analyses were performed using the SPSS 19.0 software (Chicago, IL, USA). Values of $p<0.05$ were considered statistically significant.

\section{Results}

Effect of SE on APAP-induced acute toxicity as assessed through evaluation of hepatic function markers

In an initial animal experiment, we analyzed plasma ALT and AST activities (Fig. 1), which are markers of liver injury and dysfunction. Administration of APAP led

Table 2 Oligonucleotide primer sequences and PCR conditions for real-time RT-PCR

\begin{tabular}{llll}
\hline Gene (accession no.) & Primer sequences & & PCR product length (bp) \\
\cline { 2 - 4 } & Sequence (5' to 3') & GAC ACC TCA CTG AAT GGC TTC & 103 \\
\hline Cyp1a2 & Forward & ACA CAA AGG GGT CTT TCC ACT G & 91 \\
Cyp2e1 & Reverse & CAT TCC TGT GTT CCA GGA GTA CAA G & \\
(NM_021282) & Forward & GAT ACT TAG GGA AAA CCT CCG CAC & 98 \\
GAPDH & Reverse & TGG TGA AGG TCG GTG TGA AC & \\
(NM_008084) & Forward & GTC GTT GAT GGC AAC AAT CTC C & \\
\hline
\end{tabular}



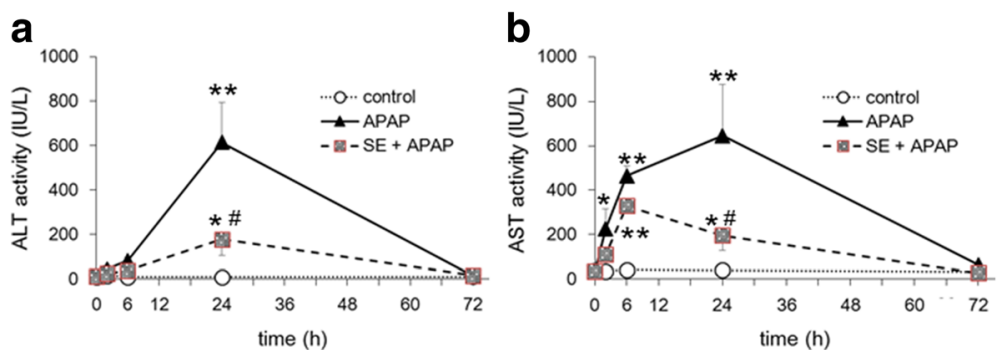

Fig. 1 Effect of pretreatment with SE on levels of hepatic injury markers. Mice received SE or vehicle (saline) by oral gavage once daily for a week. Twenty-four hours after final SE administration, $550 \mathrm{mg} / \mathrm{kg}$ of APAP was injected intraperitoneally. Mice were fasted for $16 \mathrm{~h}$ before APAP injection. Plasma levels of hepatic injury markers were determined 2, 6, 24, and $72 \mathrm{~h}$ after administration of APAP. Panels $\mathbf{a}$ and $\mathbf{b}$ indicate ALT and AST, respectively. Data are plotted as mean \pm SD for groups of six mice each. Single asterisk indicates $p<0.05$ versus control group, double asterisk indicates $p<0.01$ versus control group, and number sign indicates $p<0.05$ versus APAP group

to a slight increase in ALT activity at 2 and $6 \mathrm{~h}$ and a significant increase at $24 \mathrm{~h}$ (Fig. 1a). These levels decreased at $72 \mathrm{~h}$. APAP increased AST activity beginning at $2 \mathrm{~h}$, with the highest activity observed at $24 \mathrm{~h}$ (Fig. 1b). In contrast, pretreatment with $\mathrm{SE}$ attenuated APAPinduced ALT and AST activities at 24 h.

\section{Changes in hepatic MDA and GSH levels in APAP-exposed mice pretreated with SE}

Along with the measurement of liver function markers, we determined hepatic MDA level as a marker of lipid peroxidation. APAP treatment significantly increased MDA levels at 2, 6, and $24 \mathrm{~h}$, as expected, and pretreatment with SE attenuated APAP-induced MDA upregulation at $24 \mathrm{~h}$ (Fig. 2a). We also measured hepatic GSH content, since APAP is well known to deplete hepatic GSH [16]. APAP exposure completely depleted hepatic GSH at $2 \mathrm{~h}$ and slightly recovered at $6 \mathrm{~h}$. In addition, the GSH levels recovered beginning at $24 \mathrm{~h}$ (Fig. 2b). Although pretreatment with SE significantly decreased GSH levels compared to those observed for control, the depletion rate was significantly lower than that of the APAP group at all time we tested.

\section{Influence of SE on APAP-induced acute toxicity as assessed through evaluation of plasma TNFa levels}

Exposure to APAP is known to elevate inflammatory responses [19]. In the present study, we measured plasma TNF $\alpha$ as a representative inflammatory cytokine (Fig. 3a). Our results showed that APAP treatment significantly upregulated hepatic plasma TNFo levels, which were significantly decreased by pretreatment with SE. Moreover, in parallel with plasma TNF $\alpha$, we also investigated hepatic TNF $\alpha$ production by immunostaining since the liver is the main target of APAP-induced toxicity. We observed hepatic TNFo-production by APAP exposure (Fig. 3c). In addition, pretreatment with SE prevented APAP-induced inflammatory response in the liver (Fig. 3d).

\section{Effect of SE against APAP-induced acute toxicity as assessed through evaluation of hepatic structure} To further investigate the protective effect of SE against APAP-induced hepatotoxicity, we conducted
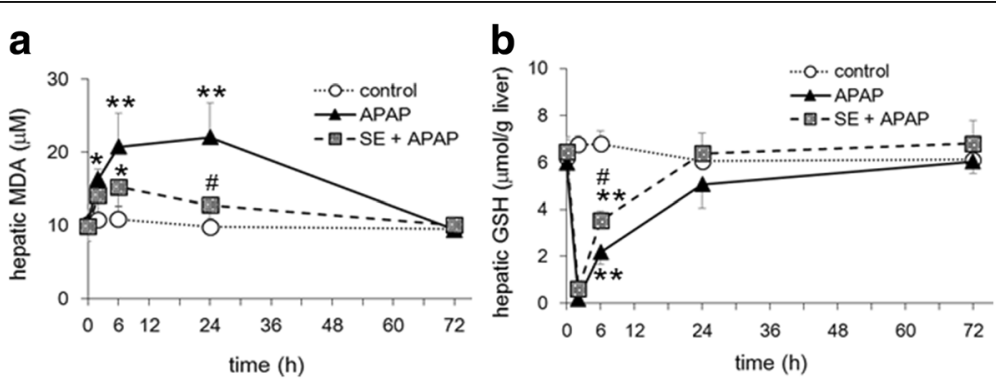

Fig. 2 Pretreatment with SE counteracts the effects of acute APAP-induced toxicity on MDA and GSH levels. Animals treated as described in Fig. 1 were euthanized 2, 6, 24, and $72 \mathrm{~h}$ post-intraperitoneal injection, and the livers were harvested at necropsy. Liver specimens were assessed for MDA levels (a) and GSH levels (b). Data are plotted as mean \pm SD for groups of six mice each. Single asterisk indicates $p<0.05$ versus control group, double asterisk indicates $p<0.01$ versus control group, and number sign indicates $p<0.05$ versus APAP group 

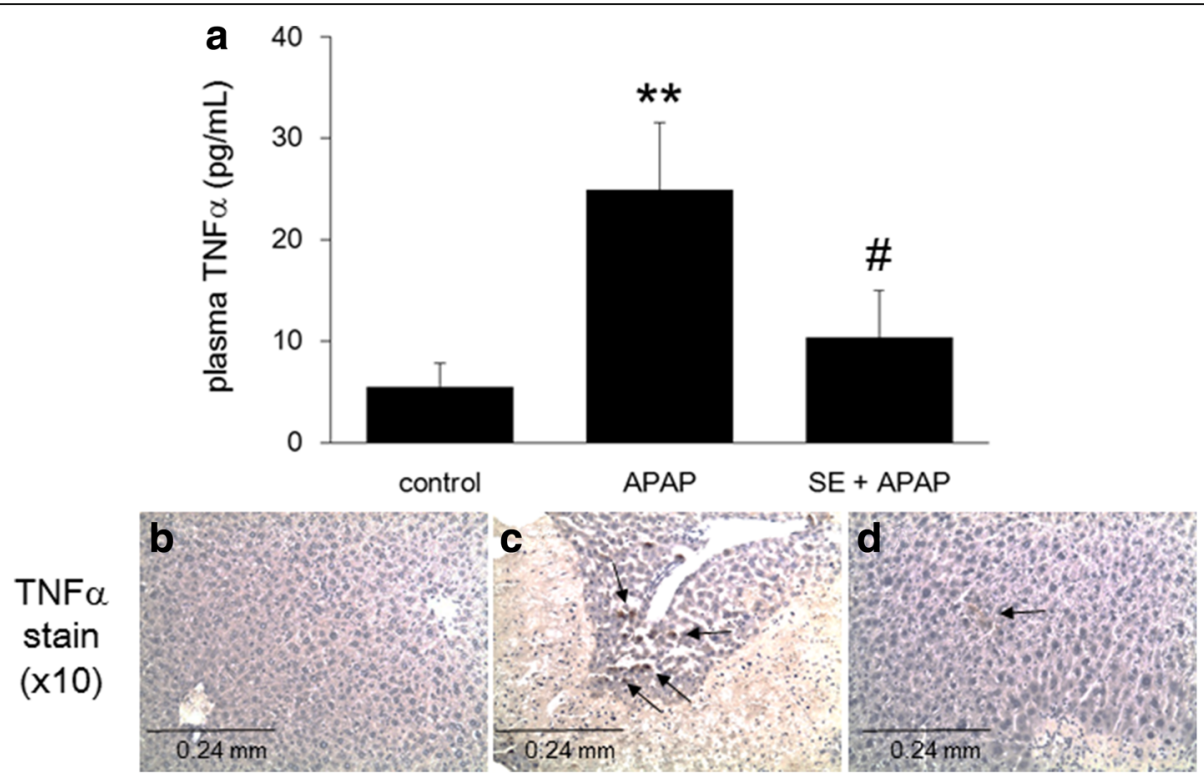

Fig. 3 Pretreatment with SE counteracts the inflammatory response induced by acute APAP-induced toxicity. Animals treated as in Fig. 1 were euthanized at $24 \mathrm{~h}$ post-intraperitoneal injection, and plasma TNFa levels (a) were determined. Data are plotted as mean \pm SD of groups of six mice each. Double asterisk indicates $p<0.01$ versus control group, and number sign indicates $p<0.05$ versus APAP group. Liver specimens were fixed and processed, and sections were stained with TNFa antibody. These micrographs provide $\times 10$ magnified images of representative TNFa-stained sections of the livers obtained from control (b), APAP (c), and SE + APAP (d) group animals. Black arrows indicate TNFa production

histopathological studies (Fig. 4). Liver sections stained with H\&E showed normal cell morphology, well-preserved cytoplasm, and a clear, plump nucleus in the control groups (Fig. 4a, d). However, in APAPinjected mice, severe necrosis and apoptosis were observed (Fig. 4b, e). Although mice pretreated with SE showed necrosis around the central vein $(\mathrm{CV})$, the necrotic area was lower than that in the APAPexposed group (Fig. 4c, f). Moreover, we investigated the protective effect of SE on nuclear DNA fragmentation and DNA strand breaks (TUNEL assay, Fig. 5). The TUNEL assay showed extensive DNA damage in cells around CV in APAP-exposed animals (Fig. 5b, e). In addition, nuclear DNA fragment was also observed

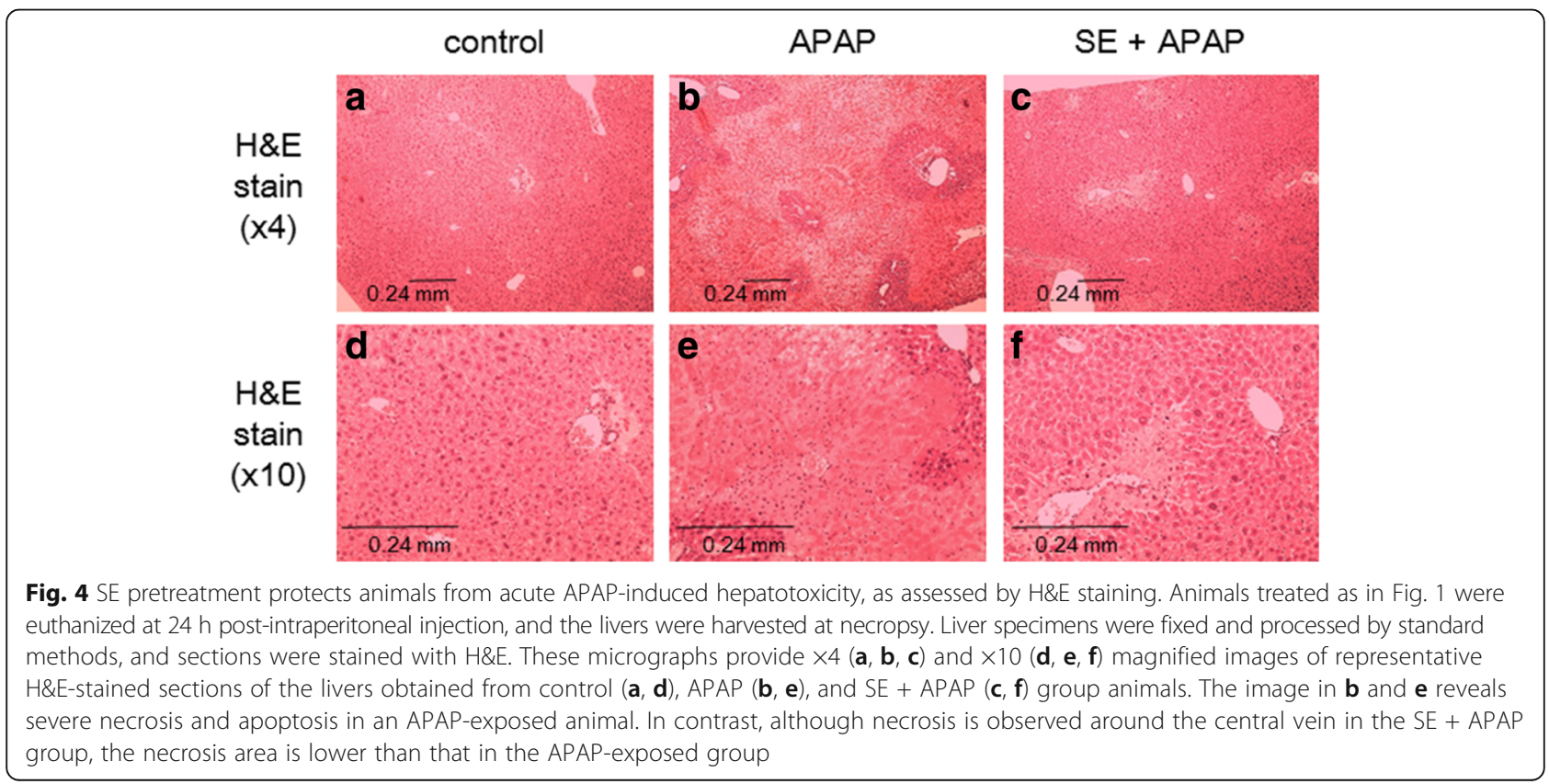




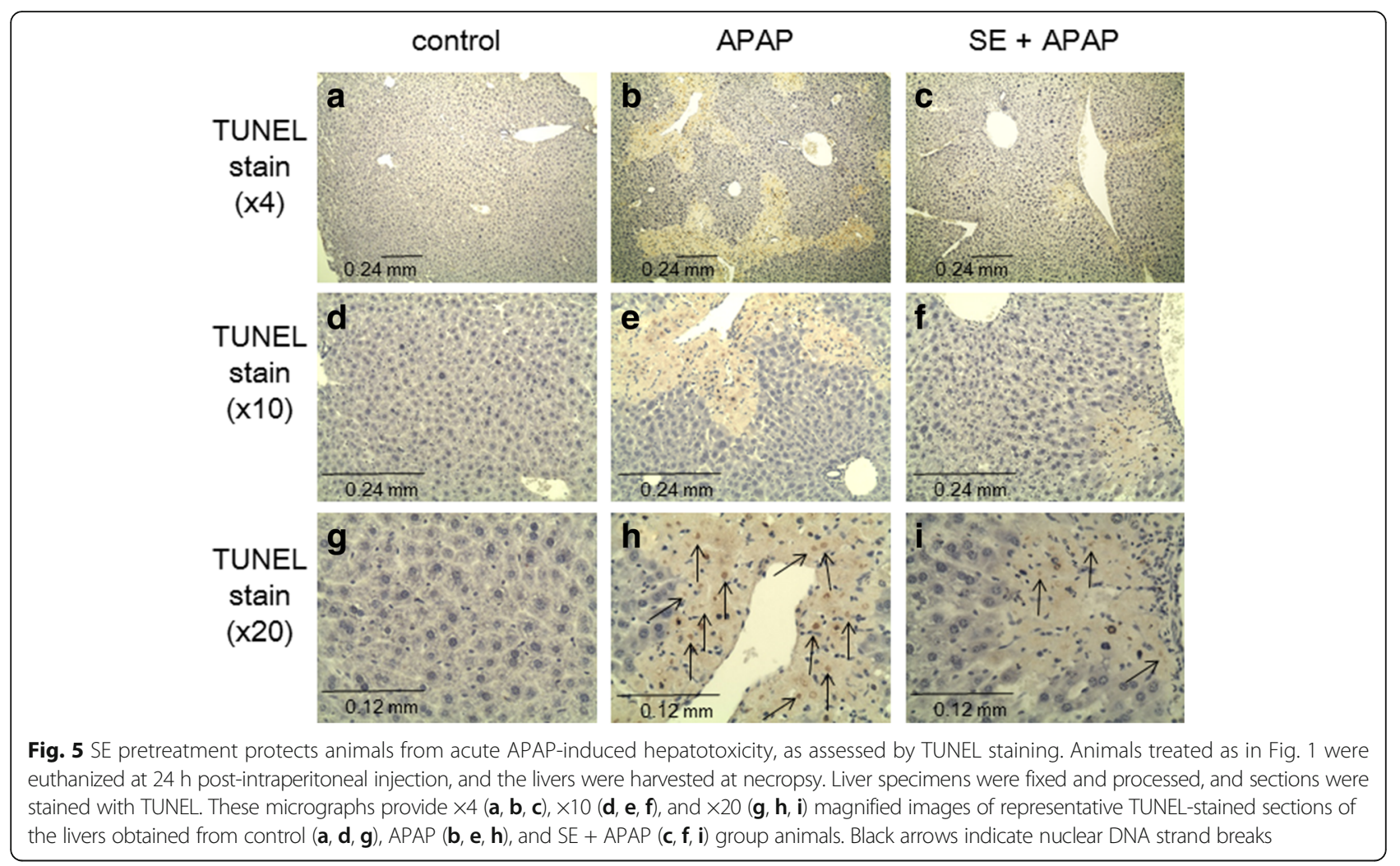

(Fig. 5h). In contrast, pretreatment with SE attenuated nuclear DNA fragmentation and DNA strand breaks, respectively (Fig. 5c, f, i).

\section{Protective effect of SE through JNK and RIP inactivation} According to several reports, JNK activation plays a key role in APAP-induced hepatic injury [29-31]. Moreover, APAP-induced hepatic injury is thought to involve primarily hepatocyte necrosis, and RIP1 is emerging as a key regulator of necrotic cell death [32, 33]. We examined the protective effect of SE on JNK and RIP1 activation in APAP-exposed mouse (Fig. 6). As expected, APAP significantly increased the level of hepatic phosphorylated JNK and RIP1 activation. In addition, APAP-induced hepatic JNK phosphorylation and RIP1 activation were attenuated by pretreatment with SE. Moreover, we observed hepatic Cyp2e1 level. Although APAP group and SE + APAP groups indicated a tendency of Cyp2e1 decrease, these two groups in Cyp2e1 level were comparable.

\section{Protective effect of SE through evaluation of Cyp induction and antioxidant capacity}

To further investigate the protective effect of SE against APAP-induced hepatotoxicity, we measured hepatic Cyp1a2 and Cyp2e1 mRNA expression (Fig. 7a, b). SE treatment decreased Cyp1a2 expression and significantly decreased Cyp2e1 mRNA. In addition, the same tendency was also observed in Cyp2e1 protein levels

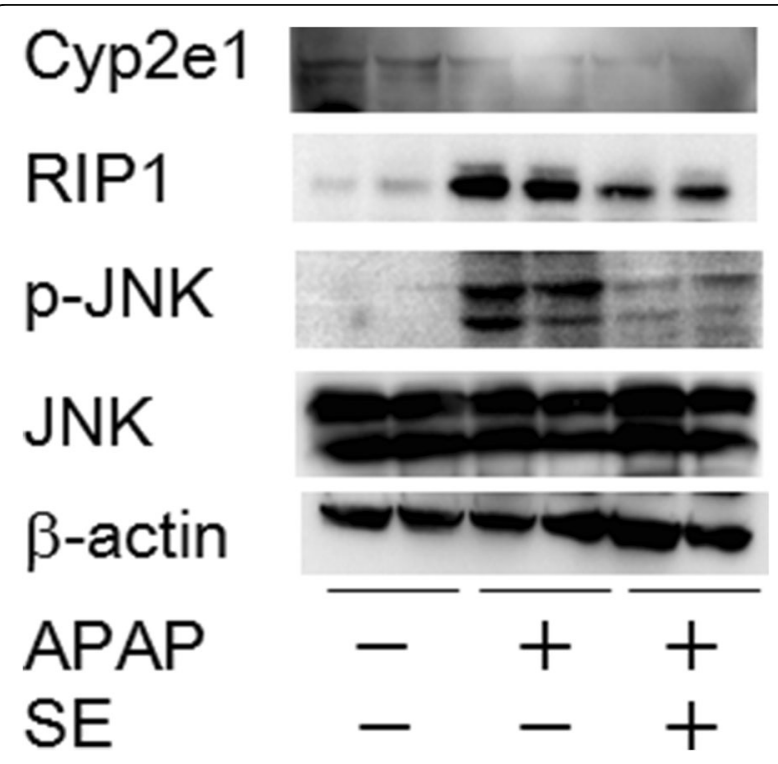

Fig. 6 Protective effect of SE through JNK and RIP inactivation. Animals treated as in Fig. 1 were euthanized at $24 \mathrm{~h}$ post-intraperitoneal injection. The livers were harvested, and proteins were collected. Data indicate hepatic Cyp2e1, RIP1, phosphorylated JNK, and JNK activation, respectively. As internal control, we measured $\beta$-actin 


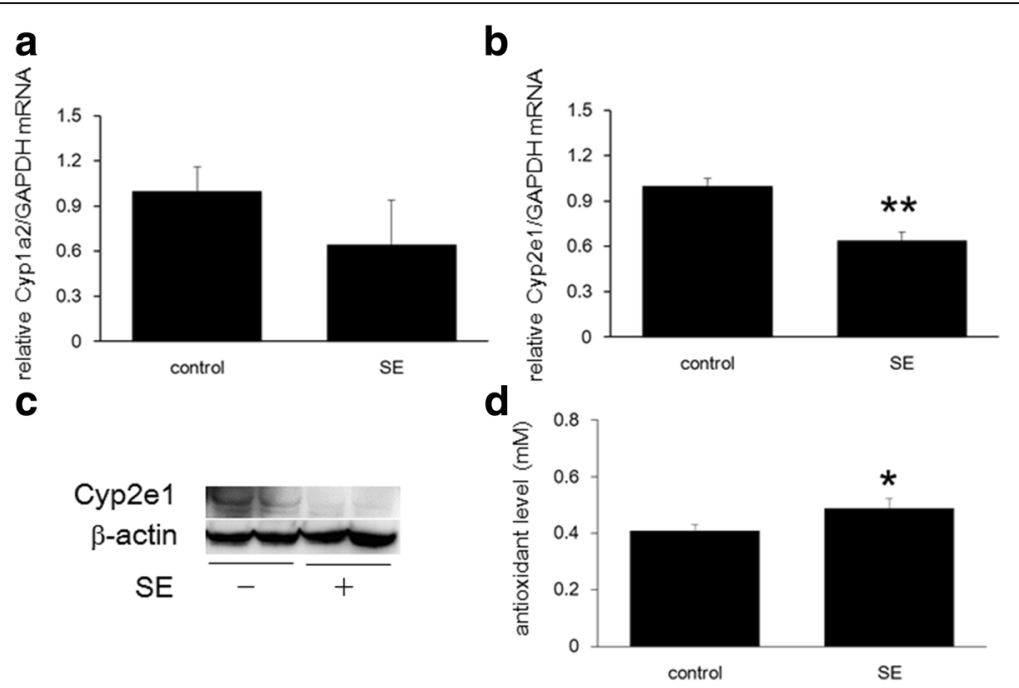

Fig. 7 Cyp induction and antioxidant effect of SE. Mice received SE or vehicle (saline) by oral gavage once daily for a week. Twenty-four hours after final pretreatment, the livers were harvested at necropsy. Panels $\mathbf{a}$ and $\mathbf{b}$ indicate Cypla2 and Cypzel mRNA levels, respectively. Panel $\mathbf{d}$ indicates hepatic antioxidant capacity. Data are plotted as mean \pm SD of groups of six mice each. Single asterisk indicates $p<0.05$, and double asterisk indicates $p<0.01$ versus control group. Data in $\mathbf{c}$ indicate hepatic Cyp2e1 protein levels. As internal control, we measured $\beta$-actin

(Fig. 7c). These data suggest that SE modulates Cyp expression.

We also studied the effect of SE on hepatic antioxidant capacity (Fig. 7d). Total antioxidant capacity was significantly increased by SE treatment, indicating that SE has significant antioxidant activity.

\section{Discussion}

This study demonstrated that pretreatment with SE can prevent APAP overdose-induced acute toxicity, as assessed by evaluating blood function markers, oxidative stress (MDA and GSH level), inflammatory responses, JNK activation, and RIP1 activation in the liver. In addition, SE also prevented APAP-induced necrotic and apoptotic cell death, respectively. Although apoptosis occurred by APAP treatment, ratio of TUNEL-positive cells was lower compared with necrosis area. Moreover, TUNEL-positive nucleus is observed within the area of necrosis. That suggests TUNEL-positive cells in APAPinduced cell death reflect necrosis-mediated DNA fragmentation not apoptotic cell death because caspase-3, that is well known to apoptosis marker, was not elevated by APAP treatment $[34,35]$.

In the present study, although we cannot show whether TUNEL-positive cells were necrosis-mediated DNA fragmentation or apoptotic cell death, these are consistent with past literature that APAP-induced liver injury results from mainly necrotic rather than apoptotic cell death [34, 36].

Since APAP-induced hepatotoxicity occurs in multiple steps, SE may have protective effects against several steps. Initially, we hypothesized that SE acts as an antioxidant. Oxidative stress is a mechanism that has been postulated to be important in the development of APAP-induced hepatotoxicity [16, 37, 38]. Numerous studies have reported that antioxidants can prevent hepatic damage by counteracting free radicals and preventing lipid peroxidation $[39,40]$. We found that pretreatment of APAP-treated mice with SE reduced levels of hepatic MDA, a naturally occurring product of lipid peroxidation. Several literature reports have hypothesized that SE exhibits antioxidant activity because many new flavonoids and polyphenols have been discovered in S. veitchii [41, 42]. Our hypothesis is supported by the well-known antioxidant properties of such compounds [43, 44], as well as our observation of SEinduced increase in hepatic antioxidant capacity. Chlorophyll might also be a candidate active molecule since it has demonstrated antioxidant activity and is present in abundance $(250 \mathrm{mg} / \mathrm{mL})$. Further, Serpeloni et al. [45] have demonstrated that 13-day oral treatment with chlorophyll (0.5 mg/kg) suppressed cisplatin-induced oxidative stress in mice. Although the period of SE administration was shorter in our study, the chlorophyll content in our SE was much greater than $0.5 \mathrm{mg}$ per day. Therefore, the chlorophyll in SE could have contributed to the suppression of APAP-induced oxidative stress in our study.

In clinical practice, NAC has been used to treat patients with APAP overdose since it has the potential to promote hepatic GSH synthesis [46] and APAP is well known to deplete GSH [47]. Moreover, GSH injection is effective in mice. However, GSH upregulation is not observed upon pretreatment with SE. Taken together, 
our data indicate that SE may protect against APAP toxicity by ameliorating the impairment of antioxidant systems except for GSH.

Another possible explanation for our results is that the protective effect of SE against APAP-induced toxicity may possibly reflect inhibition of some CYPs, which would in turn prevent the formation of toxic NAPQI. Cyp2e1 gene knockout mouse or Cyp2e1 inhibition by an inhibitor such as disulfiram or natural product such as resveratrol and schisandra attenuated APAP-induced hepatotoxicity in mice [48-50]. In addition, Cyp1a2 knockout mice showed reduced APAP-induced toxicity $[51,52]$. We used qRT-PCR to investigate the effect of SE on Cyp2e1 and Cyp1a2 mRNA expression and showed that SE significantly decreased Cyp2e1 gene expression. Furthermore, the same tendency was also observed in Cyp2e1 protein levels. These might provide an additional explanation of the protective mechanism of SE against APAP toxicity. In addition, we observed hepatic Cyp2e1 level $24 \mathrm{~h}$ after APAP treatment. Although APAP group and SE + APAP groups indicated a tendency of Cyp2e1 decrease, these two groups in Cyp2e1 level were comparable. Several literatures reported Cyp2e1 was degraded after administration of toxic doses of APAP, possibly through interaction between Cyp2e1 and NAPQI or other metabolites produced from Cypsmediated metabolism of APAP. Our data was consistent with these reports $[53,54]$. On the other hand, in SE + APAP group, we cannot prove Cyp2e1 decrease was whether depending on APAP-induce degradation or inhibition potential by SE. Further investigation is needed to elucidate this question in the future research.

An additional explanation is that SE protects against APAP-induced hepatotoxicity via its anti-inflammatory potential. It is well known that drug toxicity such as that due to APAP causes hepatocyte necrosis, which results in neutrophil and monocyte infiltration. It is reported that Kupffer cells activate the expression of proinflammatory cytokines such as TNF $\alpha$ and IL-6 $[55,56]$. Our study also showed that APAP administration significantly induced TNF $\alpha$, which was inhibited by SE pretreatment. Nuclear factor (NF) $\mathrm{\kappa} B$ is an important transcription factor for inducible inflammatory cytokine expression [57, 58]. In vitro assays from other groups have shown that SE inhibited NF-KB activity [8], suggesting that our explanation is plausible. However, in the current study, we cannot elucidate whether repression of inflammatory response is due to direct effect or upper event such as Cyp inhibition and ROS decrease since inflammatory response is latter event in APAP-induced hepatotoxicity. Hence, further investigation might be needed to prove whether SE's anti-inflammatory potential contributes to protection against APAP-induced hepatotoxicity or not.

\section{Conclusion}

In conclusion, we demonstrated that pretreatment with SE suppresses APAP-induced hepatic injury and hypothesize that the hepatoprotective mechanisms of SE are wideranging, including inhibition of Cyp2e1 expression and antioxidant activity. Although further investigation is needed to clarify the detailed protective mechanisms and main component(s) of SE, these findings are expected to contribute to self-medication options against acute hepatic injury and disease.

\section{Acknowledgements}

The authors thank Dr. Nobuhiko Miura (Japan National Institute of Occupational Safety and Health, Japan) for his kind suggestions. We are grateful to Sunchlon Co., Ltd., for providing the SE.

\section{Funding}

This research was financially supported by Kinjo Gakuin University Research Grant.

Availability of data and materials

Please contact the author for the data requests.

\section{Authors' contributions}

HY conceived and designed the experiments; performed the experiments; analyzed and interpreted the data; contributed to the reagents, materials, analysis tools, or data; and wrote most of the paper. HU contributed to the reagents, materials, analysis tools, or data and wrote the paper in part. HF contributed to the reagents, materials, analysis tools, or data. TN performed the experiments; analyzed and interpreted the data; contributed to the reagents, materials, analysis tools, or data; and wrote the paper in part. All authors read and approved the final manuscript.

\section{Competing interests}

The authors declare that they have no competing interests.

\section{Consent for publication}

Not applicable.

Ethics approval and consent to participate

All experiments were approved by the Institutional Animal Care and Experimentation Committee of Kinjo Gakuin University (No. 129).

\section{Publisher's Note}

Springer Nature remains neutral with regard to jurisdictional claims in published maps and institutional affiliations.

\section{Author details}

${ }^{1}$ College of Pharmacy, Kinjo Gakuin University, 2-1723 Omori, Moriyamaku, Nagoya, Aichi 463-8521, Japan. ${ }^{2}$ Department of Pharmacology, Shimane University Faculty of Medicine, 89-1 Enya-cho, Izumo, Shimane 693-8501, Japan.

Received: 3 February 2017 Accepted: 4 June 2017

Published online: 12 June 2017

References

1. Kweon MH, Hwang HJ, Sung HC. Identification and antioxidant activity of novel chlorogenic acid derivatives from bamboo (Phyllostachys edulis). J Agric Food Chem. 2001;49:4646-55.

2. Wang J, Yue YD, Jiang H, Tang F. Rapid screening for flavone C-glycosides in the leaves of different species of bamboo and simultaneous quantitation of four marker compounds by HPLC-UV/DAD. Int J Anal Chem. 2012;2012:205101.

3. Park HS, Lim JH, Kim HJ, Choi HJ, Lee IS. Antioxidant flavone glycosides from the leaves of Sasa borealis. Arch Pharm Res. 2007:30:161-6.

4. Seki T, Maeda H. Cancer preventive effect of Kumaizasa bamboo leaf extracts administered prior to carcinogenesis or cancer inoculation. Anticancer Res. 2010;30:111-8. 
5. Seki T, Kida K, Maeda H. Immunostimulation-mediated anti-tumor activity of bamboo (Sasa senanensis) leaf extracts obtained under 'vigorous' condition. Evid Based Complement Alternat Med. 2010;7:447-57.

6. Okada Y, Okajima H, Takeshita K, Kanamori M. Kinetic study of Sasa veitchii extract as a radical scavenger and an antioxidant. J Food Sci. 2012;77:C1211-7.

7. Hayashi K, Lee J-B, Kurosaki Y, Nozawa M, Asai S, Takeshita K, et al. Evaluation of fractions and isolated polysaccharides from Sasa veitchii for their preventive effects on influenza A virus infection. J Funct Foods. 2014;10:25-34.

8. Van Hoyweghen L, De Bosscher K, Haegeman G, Deforce D, Heyerick A. In vitro inhibition of the transcription factor NF-kappaB and cyclooxygenase by bamboo extracts. Phytother Res. 2014;28:224-30.

9. Usuda H, Fujii H, Nonogaki T. Sasa veitchii extracts suppress 2,4dinitrofluorobenzene-induced contact hypersensitivity in mice. Food Agric Immunol. 2016;27:523-34.

10. Ma JQ, Ding J, Zhang L, Liu CM. Hepatoprotective properties of sesamin against CCl4 induced oxidative stress-mediated apoptosis in mice via JNK pathway. Food Chem Toxicol. 2014;64:41-8

11. Bhondave PD, Devarshi PP, Mahadik KR, Harsulkar AM. 'Ashvagandharishta' prepared using yeast consortium from Woodfordia fruticosa flowers exhibit hepatoprotective effect on $\mathrm{CCl} 4$ induced liver damage in Wistar rats. J Ethnopharmacol. 2014:151:183-90.

12. Yoshioka H, Tanaka M, Fujii H, Nonogaki T. Sasa veitchii extract suppresses carbon tetrachloride-induced hepato- and nephrotoxicity in mice. Environ Health Prev Med. 2016;21:554-62.

13. Weber LW, Boll M, Stampfl A. Hepatotoxicity and mechanism of action of haloalkanes: carbon tetrachloride as a toxicological model. Crit Rev Toxicol. 2003;33:105-36.

14. Yoshioka H, Fukaya S, Onosaka S, Nonogaki T, Nagatsu A. Kampo formula "Hochu-ekki-to" suppressed carbon tetrachloride-induced hepatotoxicity in mice. Environ Health Prev Med. 2016;21:579-84.

15. McGill MR, Sharpe MR, Williams CD, Taha M, Curry SC, Jaeschke H. The mechanism underlying acetaminophen-induced hepatotoxicity in humans and mice involves mitochondrial damage and nuclear DNA fragmentation J Clin Invest. 2012;122:1574-83.

16. Bessems JG, Vermeulen NP. Paracetamol (acetaminophen)-induced toxicity: molecular and biochemical mechanisms, analogues and protective approaches. Crit Rev Toxicol. 2001;31:55-138.

17. Hinson JA, Roberts DW, James LP. Mechanisms of acetaminophen-induced liver necrosis. Handb Exp Pharmacol. 2010;196:369-405.

18. Abreu RV, Moraes-Santos T. The protective effect of coffee against paracetamol-induced hepatic injury in rats. J Food Biochem. 2011;35:1653-9.

19. Krenkel O, Mossanen JC, Tacke F. Immune mechanisms in acetaminopheninduced acute liver failure. Hepatobiliary Surg Nutr. 2014;3:331-43.

20. Lee SS, Buters JT, Pineau T, Fernandez-Salguero P, Gonzalez FJ. Role of CYP2E1 in the hepatotoxicity of acetaminophen. J Biol Chem. 1996;271:12063-7.

21. Walubo A, Barr S, Abraham AM, Coetsee C. The role of cytochrome-P450 inhibitors in the prevention of hepatotoxicity after paracetamol overdose in rats. Hum Exp Toxicol. 2004:23:49-54.

22. Polson J, Lee WM. AASLD position paper: the management of acute liver failure. Hepatology. 2005;41:1179-97.

23. Smilkstein MJ, Knapp GL, Kulig KW, Rumack BH. Efficacy of oral N-acetylcysteine in the treatment of acetaminophen overdose. Analysis of the national multicenter study (1976 to 1985). N Engl J Med. 1988;319:1557-62.

24. Batista-Gonzalez AE, Silva AMDOE, Vidal-Novoa A, Pinto JR, Mancini DAP Mancini-Filho J. Analysis of in vitro and in vivo antioxidant properties of hydrophilic fractions from the seaweed Halimeda monile L. J Food Biochem. 2012;36:189-97.

25. Liu L-Y, Chang L-Y, Chou S-S, Hsiao Y-L, Chien Y-W. Studies on the antioxidant components and activities of the methanol extracts of commercially grown Hemerocallis fulva L. (daylily) in Taiwan. J Food Biochem. 2010;34:90-104.

26. Yoshioka H, Usuda H, Nonogaki T, Onosaka S. Carbon tetrachloride-induced lethality in mouse is prevented by multiple pretreatment with zinc sulfate. J Toxicol Sci. 2016:41:55-63.

27. Yoshioka H, Fukaya S, Fukuishi N, Nagatsu A, Nonogaki T, Onosaka S Bromobenzene-induced lethal toxicity in mouse is prevented by pretreatment with zinc sulfate. Chem Biol Interact. 2016;254:117-23.

28. Yoshioka H, Onosaka S. Zinc sulfate pretreatment prevents carbon tetrachloride-induced lethal toxicity through metallothionein-mediated suppression of lipid peroxidation in mice. Fundam Toxicol Sci. 2016;3:151-6.

29. Ghosh J, Das J, Manna P, Sil PC. Arjunolic acid, a triterpenoid saponin, prevents acetaminophen (APAP)-induced liver and hepatocyte injury via the inhibition of APAP bioactivation and JNK-mediated mitochondrial protection. Free Radic Biol Med. 2010;48:535-53.

30. Hanawa N, Shinohara M, Saberi B, Gaarde WA, Han D, Kaplowitz N. Role of JNK translocation to mitochondria leading to inhibition of mitochondria bioenergetics in acetaminophen-induced liver injury. J Biol Chem. 2008; 283:13565-77.

31. Beger RD, Bhattacharyya S, Yang X, Gill PS, Schnackenberg LK, Sun J, et al. Translational biomarkers of acetaminophen-induced acute liver injury. Arch Toxicol. 2015;89:1497-522

32. Vanlangenakker $\mathrm{N}$, Vanden Berghe $\mathrm{T}$, Vandenabeele P. Many stimuli pull the necrotic trigger, an overview. Cell Death Differ. 2012;19:75-86.

33. Zhang YF, He W, Zhang C, Liu XJ, Lu Y, Wang H, et al. Role of receptor interacting protein (RIP)1 on apoptosis-inducing factor-mediated necroptosis during acetaminophen-evoked acute liver failure in mice. Toxicol Lett. 2014;225:445-53.

34. Lawson JA, Fisher MA, Simmons CA, Farhood A, Jaeschke H. Inhibition of Fas receptor (CD95)-induced hepatic caspase activation and apoptosis by acetaminophen in mice. Toxicol Appl Pharmacol. 1999:156:179-86.

35. Knight TR, Jaeschke H. Acetaminophen-induced inhibition of Fas receptormediated liver cell apoptosis: mitochondrial dysfunction versus glutathione depletion. Toxicol Appl Pharmacol. 2002;181:133-41.

36. Gujral JS, Knight TR, Farhood A, Bajt ML, Jaeschke $H$. Mode of cell death after acetaminophen overdose in mice: apoptosis or oncotic necrosis? Toxicol Sci. 2002;67:322-8.

37. Jaeschke H, McGill MR, Ramachandran A. Oxidant stress, mitochondria, and cell death mechanisms in drug-induced liver injury: lessons learned from acetaminophen hepatotoxicity. Drug Metab Rev. 2012;44:88-106.

38. Chen $Y$, Dong $H$, Thompson DC, Shertzer HG, Nebert DW, Vasiliou V. Glutathione defense mechanism in liver injury: insights from animal models. Food Chem Toxicol. 2013;60:38-44.

39. Ramachandran A, Lebofsky M, Baines CP, Lemasters JJ, Jaeschke H. Cyclophilin D deficiency protects against acetaminophen-induced oxidant stress and liver injury. Free Radic Res. 2011:45:156-64.

40. McGill MR, Williams CD, Xie Y, Ramachandran A, Jaeschke $H$. Acetaminophen-induced liver injury in rats and mice: comparison of protein adducts, mitochondrial dysfunction, and oxidative stress in the mechanism of toxicity. Toxicol Appl Pharmacol. 2012;264:387-94.

41. Zhang Y, Jiao J, Liu C, Wu X, Zhang Y. Isolation and purification of four flavone C-glycosides from antioxidant of bamboo leaves by macroporous resin column chromatography and preparative high-performance liquid chromatography. Food Chem. 2008;107:1326-36.

42. Zulkafli ZD, Wang H, Miyashita F, Utsumi N, Tamura K. Cosolvent-modified supercritical carbon dioxide extraction of phenolic compounds from bamboo leaves (Sasa palmata). J Supercrit Fluids. 2014;94:123-9.

43. Pietta PG. Flavonoids as antioxidants. J Nat Prod. 2000;63:1035-42.

44. Scalbert A, Johnson IT, Saltmarsh M. Polyphenols: antioxidants and beyond. Am J Clin Nutr. 2005;81:215S-7S.

45. Serpeloni JM, Grotto D, Aissa AF, Mercadante AZ, Bianchi Mde L, Antunes LM. An evaluation, using the comet assay and the micronucleus test, of the antigenotoxic effects of chlorophyll b in mice. Mutat Res. 2011;725:50-6.

46. Lauterburg BH, Corcoran GB, Mitchell JR. Mechanism of action of Nacetylcysteine in the protection against the hepatotoxicity of acetaminophen in rats in vivo. J Clin Invest. 1983;71:980-91.

47. Saito C, Zwingmann C, Jaeschke H. Novel mechanisms of protection against acetaminophen hepatotoxicity in mice by glutathione and $\mathrm{N}$-acetylcysteine. Hepatology. 2010:51:246-54.

48. Zaher H, Buters JT, Ward JM, Bruno MK, Lucas AM, Stern ST, et al. Protection against acetaminophen toxicity in CYP1A2 and CYP2E1 double-null mice. Toxicol Appl Pharmacol. 1998;152:193-9.

49. Jiang $Y$, Fan $X$, Wang $Y$, Tan $H$, Chen $P$, Zeng $H$, et al. Hepato-protective effects of six schisandra lignans on acetaminophen-induced liver injury are partially associated with the inhibition of CYP-mediated bioactivation. Chem Biol Interact. 2015:231:83-9.

50. Wang $Y$, Jiang $Y$, Fan $X$, Tan $H$, Zeng $H$, Chen $P$, et al. Hepato-protective effect of resveratrol against acetaminophen-induced liver injury is associated with inhibition of CYP-mediated bioactivation and regulation of SIRT1-p53 signaling pathways. Toxicol Lett. 2015;236:82-9.

51. Tonge RP, Kelly EJ, Bruschi SA, Kalhorn T, Eaton DL, Nebert DW, et al. Role of CYP1A2 in the hepatotoxicity of acetaminophen: investigations using Cyp1a2 null mice. Toxicol Appl Pharmacol. 1998;153:102-8.

52. Hazai E, Vereczkey L, Monostory K. Reduction of toxic metabolite formation of acetaminophen. Biochem Biophys Res Commun. 2002;291:1089-94. 
53. Snawder JE, Roe AL, Benson RW, Roberts DW. Loss of CYP2E1 and CYP1A2 activity as a function of acetaminophen dose: relation to toxicity. Biochem Biophys Res Commun. 1994;203:532-9.

54. Sinclair JF, Szakacs JG, Wood SG, Kostrubsky VE, Jeffery EH, Wrighton SA, et al. Acetaminophen hepatotoxicity precipitated by short-term treatment of rats with ethanol and isopentanol: protection by triacetyloleandomycin. Biochem Pharmacol. 2000;59:445-54.

55. Martin-Murphy BV, Holt MP, Ju C. The role of damage associated molecular pattern molecules in acetaminophen-induced liver injury in mice. Toxicol Lett. 2010;192:387-94.

56. He CY, Liang BB, Fan XY, Cao L, Chen R, Guo YJ, et al. The dual role of osteopontin in acetaminophen hepatotoxicity. Acta Pharmacol Sin. 2012:33:1004-12.

57. Oeckinghaus A, Hayden MS, Ghosh S. Crosstalk in NF-kappaB signaling pathways. Nat Immunol. 2011;12:695-708.

58. Shen H, Sheng L, Chen Z, Jiang L, Su H, Yin L, et al. Mouse hepatocyte overexpression of NF-kappaB-inducing kinase (NIK) triggers fatal macrophagedependent liver injury and fibrosis. Hepatology. 2014;60:2065-76.

Submit your next manuscript to BioMed Central and we will help you at every step:

- We accept pre-submission inquiries

- Our selector tool helps you to find the most relevant journal

- We provide round the clock customer support

- Convenient online submission

- Thorough peer review

- Inclusion in PubMed and all major indexing services

- Maximum visibility for your research

Submit your manuscript at www.biomedcentral.com/submit
Biomed Central 